\title{
Refined association of TSH receptor susceptibility locus to Graves' disease in the Chinese Han population
}

\author{
Bing-Li Liu*, Shao-Ying Yang*, Wei Liu, Li-Qiong Xue, Xia Chen ${ }^{1}$, Chun-Ming Pan, \\ Zhao-Hui Gu, Ming Zhan, Xiao-Mei Zhang ${ }^{2}$, Jun Liang ${ }^{3}$, Guan-Qi Gao ${ }^{4}$, Wen-Hua Du${ }^{4}$, \\ Guo-Yue Yuan ${ }^{5}$, Ru Ying ${ }^{6}$, Shuang-Xia Zhao and Huai-Dong Song
}

State Key Laboratory of Medical Genomics, Shanghai Institute of Endocrinology and Metabolism, Molecular Medicine Center, Ruijin Hospital Affiliated to Shanghai Jiaotong University (SJTU) School of Medicine, Shanghai 200025, China, 'Department of Geratology, The First Affiliated Hospital of Nanjing Medical University, Nanjing, Jiangsu 210029, China, ${ }^{2}$ Department of Endocrinology, The First Hospital Affiliated to Bengbu Medical College, 287 Changhuai Road, Bengbu, Anhui Province 233004, China, ${ }^{3}$ Department of Endocrinology, The Central Hospital of Xuzhou Affiliated to Xuzhou Medical College, Xuzhou, Jiangsu Province 221009, China, ${ }^{4}$ Department of Endocrinology, The People's Hospital of Linyi, 27 Liberation Road, Linyi, Shandong Province 276003, China, ${ }^{5}$ Department of Endocrinology, The Hospital Affiliated to Jiangsu University, Zhenjiang, Jiangsu Province 212001, China and ${ }^{6}$ Department of Endocrinology, Anhui Provincial Hospital, 17 Lu Jiang Road, Hefei, Anhui Province 230001, China

*(B-L Liu and S-Y Yang contributed equally to this work)

Correspondence should be addressed to H-D Song or S-X Zhao Emails

huaidong_s1966@163.com or zhaozhao1215@gmail.com

\begin{abstract}
Background: Convincing evidence has demonstrated the association of TSH receptor (TSHR) with Graves' disease (GD) in the Chinese Han population.

Objective: The aim of this study was to identify the causal variants for GD in the region encompassing TSHR by a refining association study.

Design and methods: GD patients (1536) and 1516 sex-matched controls were recruited in the first stage, and an additional 3832 GD patients and 3426 sex-matched controls were recruited in the replication stage. Genotyping was performed using Illumina Human660-Quad BeadChips or TaqMan single nucleotide polymorphism (SNP) Genotyping Assays and the Fluidigm EP1 platform. Results: When the results of regression analysis for 74 genotyped SNPs and 922 imputed SNPs in the first-stage cohort were combined, rs179243 and rs3783949 were the probable susceptibility SNPs associated with GD in TSHR. Eleven SNPs, including rs179243 and rs3783949, were selected to further refine the association in the replication study. Finally, rs12101261 and rs179243 were confirmed as independent GD susceptibility variants in the replication and combined populations. Further, we also found that the rate of persistent TSHR autoantibody positivity $($ pTRAb + ) was significantly higher in the GD patients with the susceptible genotypes rs12101261 or rs 179243 than in the GD patients carrying the protective genotypes, after the GD patients had been treated for more than 1 year.

Conclusions: These findings indicate that rs12101261 and rs179243 are the possible causal SNPs for GD susceptibility in the TSHR gene and could serve as genetic markers to predict the outcome of pTRAb + in GD patients.
\end{abstract}

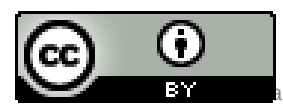

This work is licensed under a Creative Commons Attribution 3.0 Unported License. 


\section{Introduction}

Graves' disease (GD) is a common autoimmune disease with a prevalence of $\sim 1-2 \%$ in the Chinese population and is characterised by presentation with thyroidstimulating hormone receptor (TSHR) autoantibodies (TRAbs), which stimulate TSHR expression and induce hyperthyroidism. TSHR is a major thyroid-specific autoantigen and plays a key role in the regulation of thyroid function in GD. Previous studies have clearly demonstrated that GD is triggered by a combination of environmental and genetic factors. The important role of genetic factors in the pathogenesis of GD has been well established based on observation of familial clustering and evidence derived from twin studies $(1,2)$. In the past few years, studies using the strategy of candidate genes or genome-wide association studies (GWAS) have identified a few susceptibility loci for GD. The loci supported by solid evidence include HLA, CTLA4, TSHR, SCGB3A2, PTPN22 and FCRL3 $(3,4,5,6,7)$, as well as seven new loci identified by our study group, RNASET2, GDCG4p14, GPR174-ITM2A, C1QTNF6-RAC2, SLAMF6, ABO and TG $(8,9)$. RNASET2 and GDCG4p14 have been confirmed in the Caucasian GD cohorts $(10,11)$.

TSHR, on chromosome 14q31, was previously well established as a susceptibility locus for GD $(12,13,14,15$, $16,17,18,19,20)$. However, the results of studies investigating the causal variants for GD in this region have been less consistent $(21,22)$; single nucleotide polymorphisms (SNPs) in intron 7 have been suggested to be associated with GD in Japanese patients (21), but intron 1 SNPs have been found in association with GD in the Caucasian UK population $(22,23)$. Further, early studies using small samples claimed that three nonsynonymous SNPs, D36H, P52T and D727E, in TSHR were associated with GD $(12,13,14,15,16,17,18,19,20)$. In our most recent GWAS study, we have provided convincing evidence for the association of the SNPs in intron 1 of TSHR with GD in the Chinese Han population (8). In this study, we performed further fine mapping, based on our GWAS data, to identify the probable susceptibility variants for GD in TSHR. It is well-known that a positive TRAb test before discontinuation of antithyroid drugs (ATD) represents the best predictor of GD relapse $(24,25)$, suggesting that persistent TRAb positivity $(\mathrm{pTRAb}+)$ could be a marker of poor clinical outcome. In our previous report, we identified a group of SNPs in intron 1 of TSHR that are specifically associated with GD in the pTRAb-positive subgroup, but not in those without pTRAb $+(8)$. In this study, the association of susceptibility alleles in TSHR with pTRAb+ was further investigated in GD patients who had been treated with ATD for more than 1 year.

In this study, we identified two independent GD-susceptibility SNPs (rs12101261 and rs179243) by refining the association in the TSHR. The GD patients with the risk genotypes rs12101261 or rs179243 had significantly higher rates of pTRAb + than those with the protective genotypes, after treatment for more than 1 year. This indicates that rs12101261 and rs179243 may serve as genetic markers to predict the outcome of TRAb.

\section{Subjects and methods}

\section{Samples and clinical characteristics}

All participants were recruited from the Chinese Han population through collaboration with multiple hospitals in China. All subjects provided informed consent using protocols approved by the local institutional review board. GD patients (1536) and 1516 sex-matched controls were recruited in the first stage, and an additional 3994 GD patients and 3510 sex-matched controls were recruited in the replication stage $(8,9)$. Diagnosis of GD was based on documented clinical and biochemical evidence of GD, diffuse goiter, and the presence of at least one of the following: positive TRAb test, diffusely increased ${ }^{131} \mathrm{I}$ uptake in the thyroid gland, or exophthalmos $(8,9)$. Graves' eye disease was classified into six grades according to the guidelines of the American Thyroid Association. Goiters were divided into three grades according to the Common Criteria. Treatment of GD includes ATD and radioiodine (radioactive iodine, ${ }^{131} \mathrm{I}$ ). The detailed clinical information for GD patients is shown in Supplementary Table 1 , see section on supplementary data given at the end of this article. The levels of sensitive TSH, free thyroxine 3 and free triiodothyronine 4 were measured using chemiluminescence immunoassay. All individuals classified as GD were interviewed and examined by experienced clinicians. The investigation was approved by the local ethics committee.

\section{Genotyping and quality control}

In the GWAS stage, genotyping was performed using Illumina Human660-Quad BeadChips (8). Genotype clustering was conducted using Illumina BeadStudio 3.3 Software $(8,9)$. Also, the SNPs with Hardy-Weinberg equilibrium $P \leq 10^{-6}$, missing call rate $\geq 0.05$, or minor 
allele frequency (MAF) $\leq 0.01$ were removed from the association study $(8,9)$.

In the replication stage, in addition to the eight SNPs genotyped in our previous GWAS study (8), an additional three SNPs (rs179243, rs2284720 and rs3783949) were genotyped using TaqMan SNP Genotyping Assays and the Fluidigm EP1 platform. All SNPs were genotyped with a call rate of $>95 \%$ for further association analysis.

\section{Imputation}

Genotype imputation was performed using IMPUTE version 2 (http://mathgen.stats.ox.ac.uk/impute/impute _v2.html/), based on the 1000 Genomes Project (June 2011) as reference. The association analysis of the imputed SNPs was carried out using ProbABEL (26). Quality filtering was performed on SNPs before analysis to ensure robust association tests. The SNPs with Hardy-Weinberg equilibrium $P \leq 10^{-6}$, missing call rate $\geq 0.05$, or MAF $\leq 0.01$ were discarded.

\section{Statistical analysis}

GD patients (1536) and 1516 sex-matched controls were recruited in the first stage, and 1468 cases and 1490 controls remained after stringent quality control. An additional 3994 GD cases and 3510 sex-matched controls were recruited for the replication study. We successfully genotyped 11 SNPs in 3832 GD cases and 3426 controls in the replication stage (8). We analysed the SNPs from the GWAS and replication stages using the Cochran-Armitage trendtest in PLINK. In the combined stage, Cochran-MantelHaenszel stratification analysis was used to examine the associations. Forward stepwise logistic regression analysis was carried out in GWAS and the combined stage using SPSS PASW Statistics 18 packages. Conditional logistic regression analysis was performed using PLINK packages.

In stage 2, the 11 SNPs were selected using Haploview Software (http://www.broadinstitute.org/ scientific-community/science/programs/medical-andpopulation-genetics/haploview/haploview) and a pairwise-tagging approach. Haplotype association analyses, estimation of pairwise linkage disequilibrium (LD) and tagSNP selection were calculated using Haploview 4.1.

\section{Sample size and power calculations}

Sample size calculations were performed using QUANTO (version 1.2.4). Based on our GWAS data, we found that a minimum of 1343 (rs2284722) and a maximum of 3540 (rs4903964) sample pairs were needed to detect a significant difference in the 11 selected SNPs on a genome-wide significance level for each variant with allele frequencies ranging from 0.01 to 0.5 and with at least $80 \%$ power (Supplementary Table 2, see section on supplementary data given at the end of this article). In the current study, we therefore used a total of $\sim 5000$ sample pairs in the first and replication studies combined.

\section{Measurement of TRAb levels}

TRAb levels of GD patients who had been treated with ATD for more than 1 year were measured by quantitative ELISA (RSR Limited, Cardiff, UK) in our laboratory. GD patients with TRAb levels $\geq 1.5 \mathrm{U} / 1$ were defined as 'persistently TRAb-positive' and those with TRAb levels $<1.5 \mathrm{U} / 1$ were defined as 'TRAb-negative'. Interactions between SNPs of TSHR in persistently TRAb-positive or TRAb-negative GD patients were analysed using the case-only $\chi^{2}$ test in the SPSS PASW Statistics 18 package.

The association between the genotypes or alleles at rs12101261 and rs179243 and the different grades of thyroid goiter in GD patients was also analysed using the $\chi^{2}$ test in the SPSS PASW Statistics 18 package.

\section{Results}

\section{Regression analysis of GWAS-stage and imputation data}

First, a 665-kb LD block harbouring the strongest TSHR association and separated by two recombination hotspots (recombination rate $>60 \mathrm{cM} / \mathrm{Mb}$ ) located in the up- or down-streams of the LD block in the Chinese population was chosen from our GWAS data (8) for further refinement of the association (Fig. 1a). After quality control, 131 SNPs in this region, which encompasses C14orf145/TSHR, remained for the next analysis in 1468 GD patients and 1490 control subjects using the trend-test in PLINK (Supplementary Table 3, see section on supplementary data given at the end of this article and Fig. 1a). Of these SNPs, 31 showed a significance level of $P<0.001$ and 22 showed a level of $P<0.0001$.

To identify the independent susceptibility variants associated with GD in the region encompassing C14orf145/TSHR, the 31 SNPs with $P<0.001$ were analysed in the GWAS data using forward stepwise regression analysis. Interestingly, two of the 31 SNPs were independently associated with GD risk in the GWAS cohorts $\left(P_{\text {forward }}=1.94 \times 10^{-6}\right.$ for rs $2284720, P_{\text {forward }}=1.23 \times 10^{-2}$ 

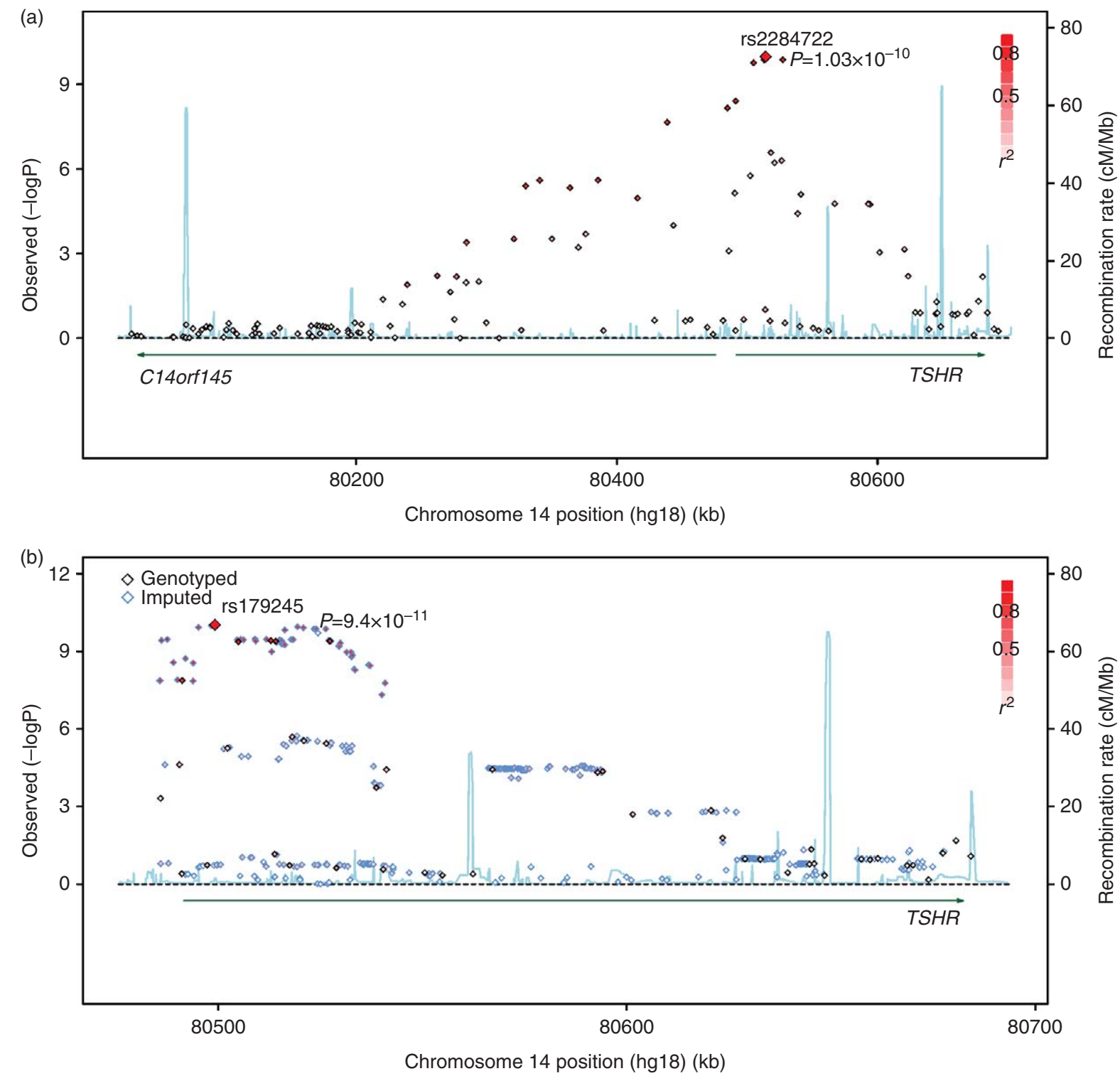

\section{Figure 1}

Regional plots of $14 q 31$ susceptibility loci associated with Graves' disease (GD) in the first stage. Values of - log10 are plotted against chromosome position, which is based on the 1000 Genomes Pilot 1. Each diamond represents one SNP, and the most strongly associated SNP is indicated by a large red diamond. The colour of each SNP block reflects its $r^{2}$ with the most strongly associated SNP within each association locus, ranging from red to white. Estimated recombination rates (based on the combined HapMap Chinese

for rs3783949). Moreover, rs2284720 improved 23 of the 30 SNP models in two-locus conditional logistic regression models (Fig. 2a). Interestingly, the seven SNP models, which were not improved by rs2284720, were improved by sample (CHB) and HapMap Japanese sample (JPT) from the HapMap project) are plotted in cyan to reflect the local LD structure around the associated SNPs. (a) Association results of genotyped SNPs on $14 q 31$ with GD in the first stage. The region plotted contains 131 SNPs covering a 665-kb region that encompassed C14orf145/TSHR in a GD case-control cohort consisting of 1468 GD cases and 1490 controls. (b) The association of genotyped and imputed SNPs in TSHR with GD is magnified in panel b.

rs3783949 (Fig. 2b). Further, the region could not be perfectly explained by separately conditioning on either rs2284720 or rs3783949; however, after conditioning on the two SNPs, no other SNPs in this region were found to be 


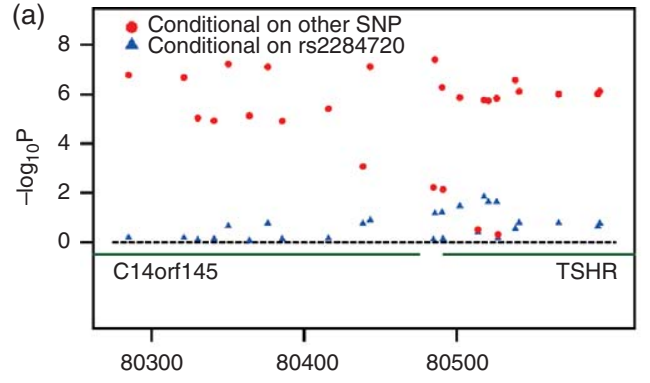

Chromosome 14 position (hg18) (kb)
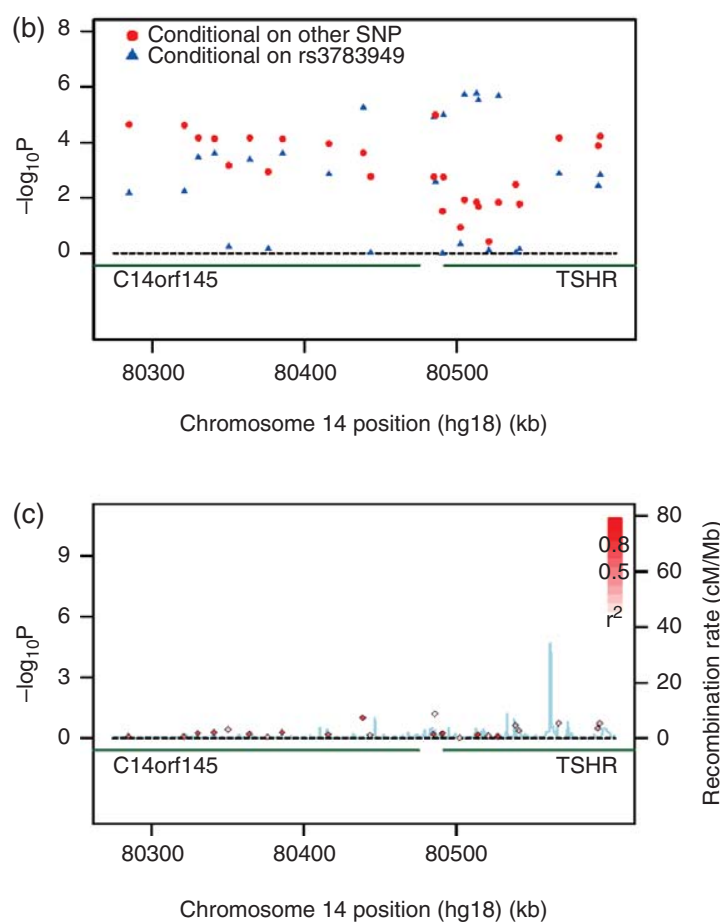

Figure 2

Results of conditional logistic regression analysis for SNPs with $P<1 \times 10^{-3}$ in $14 q 31$ in the first stage. (a and b) Two-locus conditional logistic regression for rs2284720 or rs3783949 with the other 29 genotyped SNPs with $P<1 \times 10^{-3}$. The $P$ values of the other 29 SNPs conditioning on rs2284720 or rs3783949 are shown in blue diamonds. The $P$ values of rs 2284720 or rs3783949 after conditioning on the 29 SNPs are shown in red squares. (c) After conditioning on rs2284720 and rs3783949 at the same time, the $P$ values of other SNPs in the Graves' disease susceptibility locus on $14 q 31$ are shown in panel c. None of the 29 SNPs in this susceptibility locus improved the model with rs2284720 and rs3783949 at the level of $P<0.05$. Each diamond represents one SNP. The colour of each SNP reflects its $r^{2}$ with the most strongly associated SNP, rs2284720, ranging from red to white. Estimated

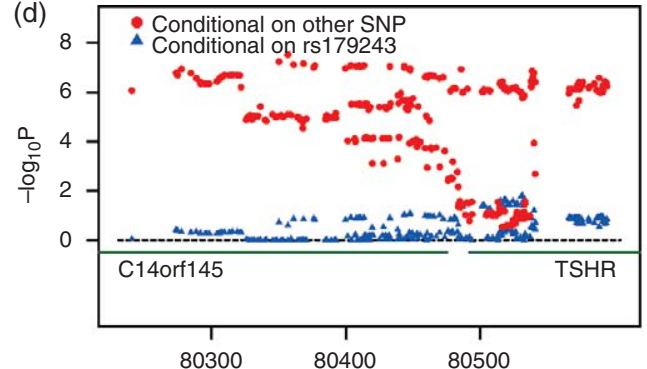

Chromosome 14 position (hg18) (kb)

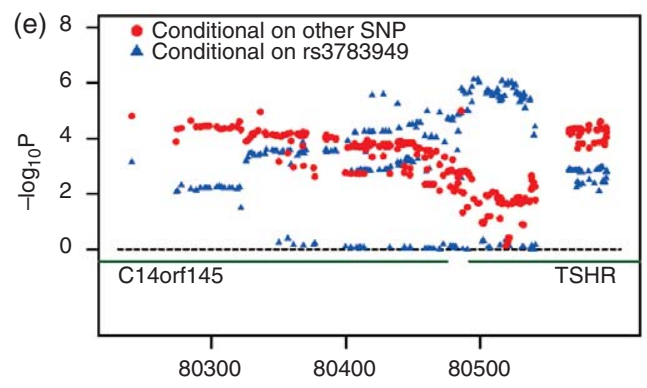

Chromosome 14 position (hg18) (kb)

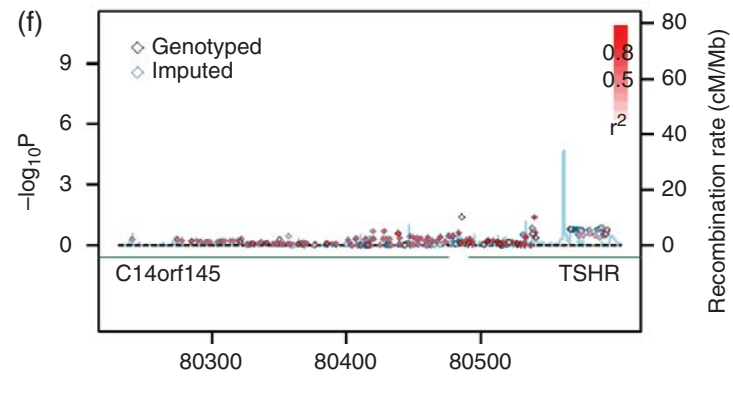

Chromosome 14 position (hg18) (kb)

recombination rates (based on the combined CHB and JPT samples from the HapMap project) are plotted in cyan to reflect the local LD structure around the associated SNPs. (d, e, and $f$ ) The results of conditional logistic regression analysis for 390 genotyped and imputed SNPs with $P<1 \times 10^{-3}$ in the GWAS samples in TSHR. (d and e) Two-locus conditional logistic regression for rs179243 or rs3783949 with SNPs other than themselves. The influences of the other SNPs on the model conditioned by rs179243 or rs3783949 are presented in blue diamonds. The $P$ values of $r$ 179243 or rs3783949 after conditioning on the other SNPs are shown in red squares. (f) After conditioning on rs179243 and rs3783949, no other SNPs in TSHR improved the model. The colour of each SNP reflects its $r^{2}$ with the most strongly associated SNP, rs179243, changing from red to white. 
independently associated with GD (Fig. 2c). Notably, rs2284720 and rs3783949 were not in a LD block $\left(r^{2}=0.15\right.$, Supplementary Figure 1, see section on supplementary data given at the end of this article) and were located in intron 1 of TSHR. These results indicate that the SNPs rs2284720 and rs3783949 in the TSHR susceptibility locus were independently associated with GD in the Chinese Han population.

Furthermore, 996 SNPs in a 443-kb region containing C14orf145/TSHR, representing an average distance of about 445 bp per SNP, were imputed based on our GWAS data using the IMPUTE 2 Software. Among the 996 SNPs, a cluster of SNPs that included rs179245 $\left(P=9.49 \times 10^{-11}\right)$, rs179243 $\left(P=1.01 \times 10^{-10}\right)$, and $\operatorname{rs} 3783943\left(P=1.14 \times 10^{-10}\right)$ located in intron 1 of TSHR showed the most significant association with GD (Supplementary Table 4, see section on supplementary data given at the end of this article and Fig. 1b). Meanwhile, the SNPs in the coding region of TSHR, P52T (rs80491931) and D727E (rs80680336), which were associated with GD in previous studies using small samples $(12,13,14,15,16,17,18,19,20)$, were not associated with GD in our study (Supplementary Table 5, see section on supplementary data given at the end of this article).

To further refine the association of candidate SNPs remaining in the $443-\mathrm{kb}$ region harbouring C14orf145/TSHR, 390 SNPs with $P<1 \times 10^{-3}$ were further analysed by two-locus logistic regression. Among the 390 SNPs, 42 SNPs containing rs2284720 and rs72693090 as the best markers for the TSHR region were individually tested in the logistic regression models, and only 18-50 SNPs improved these models with a cut-off of $P<0.05$ (Supplementary Table 6, see section on supplementary data given at the end of this article). Next, we tested a regression model, taking each one of 390 SNPs in turn, and adding each test locus. Interestingly, the majority of the markers (more than 320 SNPs in this region) were improved by the addition of each of 42 SNPs in TSHR (Supplementary Table 6). With regard to the 42 SNPs in TSHR intron 1, rs179243 probably plays the most important role in the susceptibility to GD because only 18 SNPs in this region improved the model, with rs179243 as the most strongly associated marker (Supplementary Table 6 and Fig. 2d). Interestingly, these $42 \mathrm{SNPs}$ as the best SNP predisposing to GD were perfectly linked $\left(r^{2}>0.80\right)$ (Supplementary Figure $2 \mathrm{a}$, see section on supplementary data given at the end of this article).

It was noted that 47 of the 390 SNPs in the TSHR susceptibility locus remained significantly associated with GD after conditioning on rs72693090 (Supplementary Table 6). However, the majority of models with the 47 SNPs were improved by the addition of each of another cluster of 34 SNPs in a high-LD block (Supplementary Table 7 and Figure $2 \mathrm{~b}$, see section on supplementary data given at the end of this article). Further, the models improved by 34 SNPs could not be improved by addition of these 42 SNPs, and the relatively weak association between the 34 SNPs and GD was detected in our data $\left(P\right.$ values from $2.43 \times 10^{-5}$ to $1.85 \times 10^{-6}$, Supplementary Table 7$)$. Among these 34 SNPs, the model with rs3783949 was improved by ten of 47 SNPs, and the models with each of the other 33 SNPs were improved by 16-32 SNPs (Supplementary Table 7 and Fig. 2e). Interestingly, neither rs179243 nor rs3783949 could perfectly explain the association of the SNPs in TSHR with GD (Fig. 2d and e). However, after conditioning on the two SNPs at the same time, no SNPs in TSHR were independently associated with GD at a cut-off of $P<0.05$ (Fig. 2f). These results suggest that rs179243 and rs3783949 are probably independent susceptibility loci to GD in TSHR. The results are consistent with our conclusion based on the analysis of GWAS data, because rs179243 and rs2284720 are in a complete LD block $\left(r^{2}=0.93\right)$ (Supplementary Figure 1$)$. Combing the results of logistic regression analysis of the GWAS and imputation data, it can be inferred that the SNPs rs179243 and rs3783949 are the major susceptibility variants in TSHR for GD in the Chinese Han population.

\section{Association analysis in the replication and combined populations}

The GWAS and imputation data showed that the SNPs rs179243 and rs3783949 were the major susceptibility variants in the TSHR region. These results strongly suggested the existence of two independent susceptibility SNPs to GD in the TSHR region, rs3783949 and rs179243. Therefore, in order to further refine the association of causal SNPs and GD in the TSHR region, 11 SNPs were selected for genotyping in 3994 GD cases and 3510 controls. We successfully genotyped 11 SNPs in 3832 GD cases and 3426 controls. The maximum number of sample pairs needed in the combined stage was 3540, on the basis of the results of association with GD in the first stage (Supplementary Table 2). Among the 11 SNPs, rs2284720 and rs2284722 were tightly linked to rs179243, and rs12101261 was tightly linked to rs3783949 $\left(r^{2}>0.8\right.$, Supplementary Figure 1). In addition, six tagSNPs, which can tag 13 other SNPs with $P<0.0001$ but were not in a high-LD block with rs179243 or rs3783949, were selected (Supplementary Table 8, see section on supplementary data given at the end of this article). Combining the genotyping results from GWAS and the replication stage, we confirmed the associations of the 11 SNPs with GD in 
몸 五

$\bar{u}$

ㅇํำ

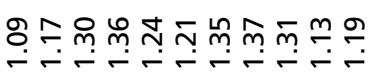

\section{응으으으으으으으응으은} $\times \times \times \times \times \times \times \times \times \times$

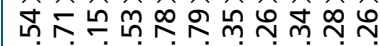
-

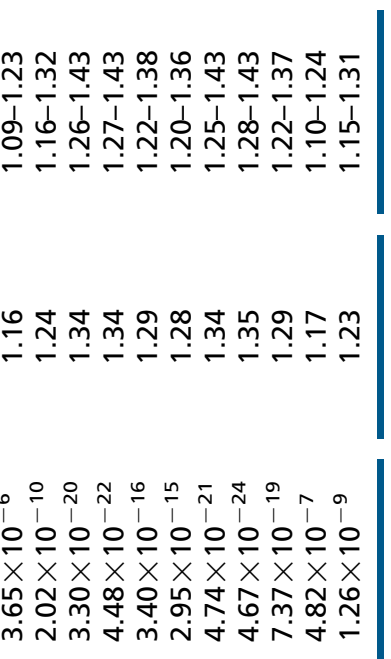

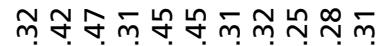

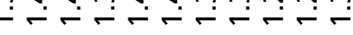

\section{ช}

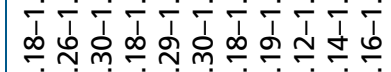

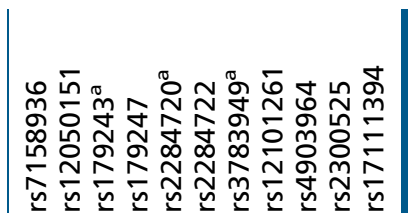

the stage 2 study, with the most significant signal at rs12101261 $\left(P_{\text {combined }}=4.67 \times 10^{-24}\right.$, Table 1$)$.

We then used forward stepwise regression analysis to identify the main-effect susceptibility variants for GD in this region. Two SNPs (rs12101261: $P_{\text {forward }}=2.22 \times 10^{-10}$; rs179243: $P_{\text {forward }}=9.61 \times 10^{-8}$ in the combined population) of the 11 SNPs were independently associated with GD in the replicated and combined cohorts (Table 2). Two-locus logistic regression analysis showed that neither rs179243 nor rs12101261 of these 11 SNPs could perfectly improve the models of the ten SNPs except themselves in TSHR (Fig. 3). However, after conditioning on the two SNPs at the same time, none of the other nine SNPs in TSHR were independently associated with GD at a cut-off $P<0.05$ (Fig. 3c and f). These results suggest that rs179243 and rs12101261 were independent susceptibility variants to GD in TSHR. It is worth mentioning that rs12101261 was tightly linked to rs3783949 $\left(r^{2}=0.93\right.$, Supplementary Figure 1$)$, which was considered an independent susceptibility tagSNP associated with GD in our GWAS and imputed data. These results indicate that there are two independent susceptibility SNPs to GD in TSHR and that the most likely independent susceptibility SNPs are rs12101261 and rs179243.

\section{The effect of the genotypes of two susceptibility SNPs in TSHR on the outcome of TRAb in GD patients}

The previous studies have revealed that a positive TRAb test before discontinuation of ATD may be considered the best predictor of GD relapse $(24,25)$, suggesting that TRAb persistence could be a marker of poor clinical outcome. The GD patients were divided into three groups according to the genotypes of rs179243 and rs12101261 respectively. The effect of the different genotypes for the two susceptibility SNPs in TSHR on the rate of persistent TRAb positivity $(\mathrm{TRAb}+)$ in GD patients after ATD treatment for more than 1 year was further investigated. After treatment, the rate of pTRAb + was higher in the GD patients with risk genotypes TT and AA at rs12101261 and rs179243, respectively, than in those with the respective, protective genotypes CC and GG (74 vs $58 \%, 75$ vs $66 \%$, $P=8.33 \times 10^{-8}$ and $6.49 \times 10^{-3}$ respectively) (Table 3 ). Further, the rate of pTRAb + was also higher in the GD patients with the heterozygous genotypes TC and AG genotypes at rs12101261 and rs179243, respectively, than in those with the respective, protective homozygous genotypes CC and GG (69 vs $58 \%, 72$ vs $66 \%, P=2.94 \times$ $10^{-4}$ and $8.12 \times 10^{-4}$ respectively) (Table 3 ). 


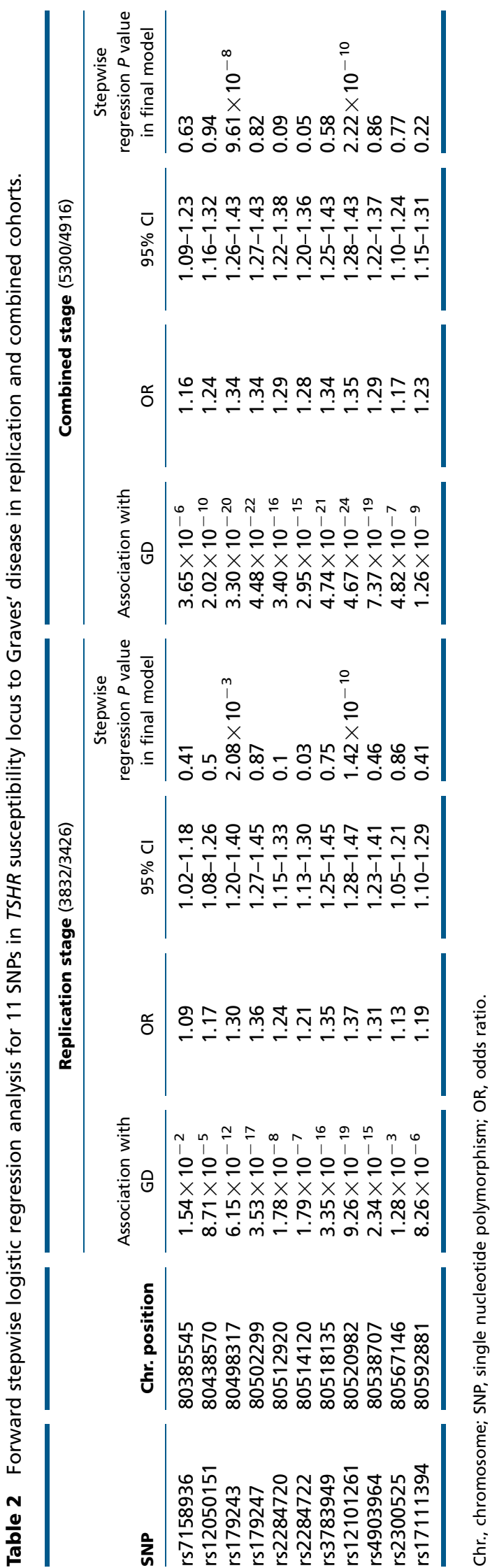

However, the risk genotypes or alleles at rs12101261 and rs179243 were not related to the different grades of thyroid goiter in GD patients (Supplementary Tables 9 and 10 , see section on supplementary data given at the end of this article).

\section{Discussion}

Although TSHR on chromosome $14 \mathrm{q} 31$ is a well-established susceptibility locus for GD, the causal variants for GD in this region have remained controversial $(21,22,23)$. In this study, we carried out a two-stage association study for 11 SNPs, including rs179243 and rs3783949 in TSHR, in a total of 5368 GD patients and 4942 sex-matched controls and found that rs12101261 and rs179243 are the two independent GD-susceptibility variants in the Chinese Han population. In addition, the rate of pTRAb + was significantly higher in GD patients with the susceptibility genotypes of rs12101261 or rs179243 than in those carrying the protective genotypes, after which the patients had been treated for more than 1 year.

In earlier studies using small sample sizes, three nonsynonymous SNPs, D36H, P52T and D727E, were claimed as susceptibility variants for $\operatorname{GD}$ in $\operatorname{TSHR}(19,27)$, but the associations have not been confirmed by subsequent studies $(12,14,16,18,22)$. In this study, we did not find an association of the nonsynonymous SNPs P52T and D727E with GD in the Chinese Han population. Moreover, the nonsynonymous $\mathrm{D} 36 \mathrm{H}$ in TSHR is a rare variant, with a MAF $<1 \%$ in the Chinese population, and was not analysed in this study. Recent case-control studies in relatively large samples suggested that SNPs in intron 7 were associated with GD in a Japanese population (21). However, on the basis of genotyping of high-density SNPs (representing an average distance of $\sim 445$ bp per SNP) in 1468 GD patients and 1490 control individuals, no SNPs in intron 7 of THSR were significantly associated with GD. In this study, a cluster of SNPs in intron 1 of TSHR were associated with GD in the Chinese Han population. The SNPs rs12101261 and rs179243 in intron 1 of TSHR were further considered as independent GD susceptibility variants in this region by fine mapping. These results are similar to those of previous reports, in which rs179247, rs2268458 and rs12101255 in intron 1 of TSHR were considered the SNPs most strongly associated with GD in a European population $(22,28,29)$. Notably, rs12101261 was perfectly linked to rs12101255 $\left(r^{2}=1.0\right)$, and rs179243 was tightly linked to rs2268458 in Europeans $\left(r^{2}=0.91\right)$. It is interesting that there were two independent GDsusceptibility SNPs in one susceptibility locus. These 
(a)

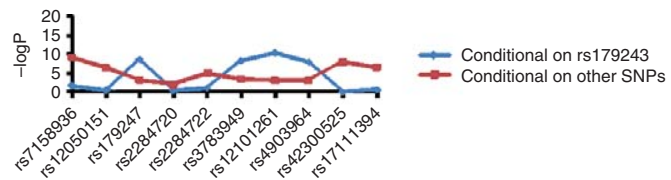

(b)

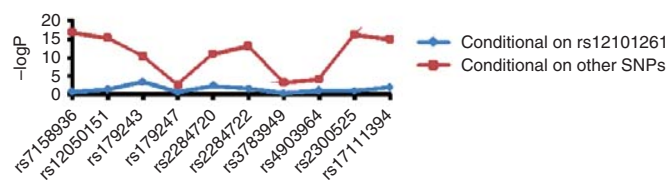

(c)

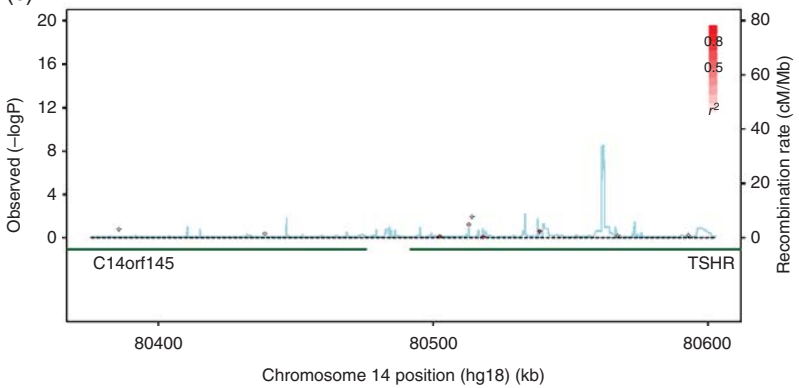

(d)

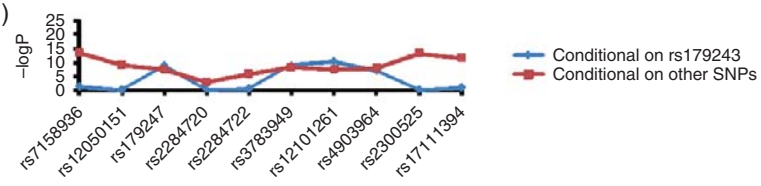

(e)

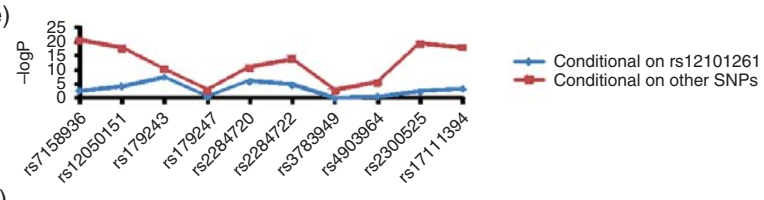

(f)

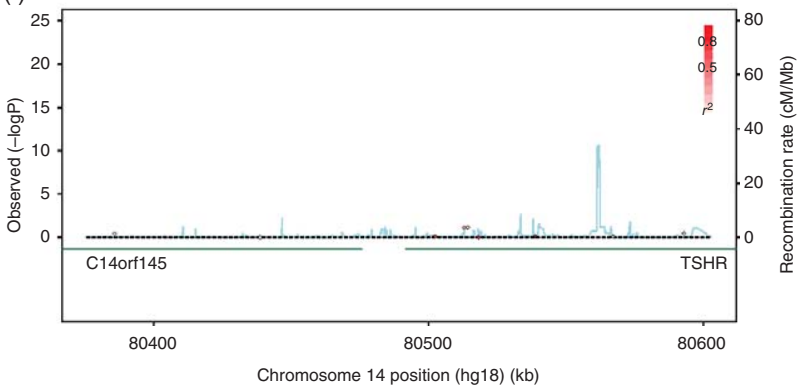

\section{Figure 3}

Results of conditional logistic regression analysis for 11 SNPs in the replication ( $a, b$, and $c$ ) and combined ( $d$, e, and f) cohorts. Two-locus conditional logistic regression analysis for $\mathrm{rs} 179243$ or rs12101261 with the other ten SNPs in replication ( $a$ and $b$ ) and combined ( $\mathrm{d}$ and e) cohorts. The $P$ values of the other SNPs conditioning on rs 179243 or rs3783949 are presented in blue diamonds. The $P$ values of rs179243 or rs12101261 after

findings indicate that the two independent GDsusceptibility SNPs rs12101261 and rs179243 in intron 1 of TSHR might be potentially involved in the development of GD when all cases are considered, but that in some patients only one of these two SNPs might be involved.

Interestingly, although the risk allele A of rs179243 has MAF in both the Chinese and Caucasian populations, the frequency of risk allele $\mathrm{T}$ at rs12101261 was conditioning on the other SNPs are presented in red squares. (c and f) After simultaneous conditioning on rs179243 and rs12101261, the other SNPs in TSHR did not significantly improve the model containing rs179243 and rs12101261 in replicated (c) and combined ( $f$ ) populations. The colour of each SNP reflects its $r^{2}$ with the most strongly associated SNP, rs12101261, changing from red to white.

dramatically different in the two ethnic populations. The frequency of the risk allele $\mathrm{T}$ at rs12101261 was $64.07 \%$ in the control individuals recruited from the Chinese Han population (8), but only $28 \%$ in healthy Caucasian Europeans (22). This finding indicates that genetic factors, such as the occurrence of rs12101261, might play an important role in the mechanism underlying the difference in GD risk variants in TSHR between Chinese

Table 3 Effect of risk genotypes at rs12101261 and rs179243 on the rate of pTRAb + in GD patients after more than 1 year of ATD treatment.

\begin{tabular}{l}
\hline SNP \\
\hline Genotype \\
\hline$P$ value \\
$\%$ TRAb $\geq 1.5$ \\
Risk genotype \\
OR \\
$95 \% \mathrm{Cl}$
\end{tabular}

\begin{tabular}{|c|c|c|}
\hline \multicolumn{3}{|c|}{ rs 12101261} \\
\hline $\mathrm{TT} / \mathrm{CT}$ & $\mathrm{CT} / \mathrm{CC}$ & $\mathrm{TT} / \mathrm{CC}$ \\
\hline $6.45 \times 10^{-3}$ & $2.94 \times 10^{-4}$ & $8.33 \times 10^{-8}$ \\
\hline 74\%/69\% & $69 \% / 58 \%$ & 74\%/58\% \\
\hline $\mathrm{TT}$ & CT & $\mathrm{TT}$ \\
\hline 1.24 & 1.59 & 1.96 \\
\hline $1.06-1.44$ & $1.23-2.04$ & $1.54-2.56$ \\
\hline
\end{tabular}

\begin{tabular}{|c|c|c|}
\hline \multicolumn{3}{|c|}{ rs179243 } \\
\hline AA/AG & AG/GG & $\mathrm{AA} / \mathrm{GG}$ \\
\hline 0.36 & $8.12 \times 10^{-4}$ & $6.49 \times 10^{-3}$ \\
\hline $75 \% / 72 \%$ & $72 \% / 66 \%$ & $75 \% / 66 \%$ \\
\hline AA & AG & AA \\
\hline 1.15 & 1.3 & 1.49 \\
\hline $0.85-1.54$ & $1.12-1.52$ & $1.12-2.00$ \\
\hline
\end{tabular}

pTRAb +, persistent TRAb-positivity; SNP, single nucleotide polymorphism; OR, odds ratio. 
and Caucasian populations. It should be pointed out that additional international collaboration studies in distinct ethnic groups will be required to further address the effects of genetic factors on the differences in GD prevalence among distinct ethnic populations.

It is well-known that patients with GD have a higher risk of relapse after ATD treatment when TRAb is persistently positive $(24,25)$. Moreover, several previous reports have revealed that a positive TRAb test before discontinuation of ATD represents the best predictor of GD relapse $(30,31)$, suggesting that TRAb persistence could be a marker of poor clinical outcome. In our most recent GWAS, we identified a group of SNPs in intron 1 of TSHR that are specifically associated with GD in the persistent TRAb-positive subgroup, but not in those without pTRAb + (8). In this study, we found that the frequencies of pTRAb + were significantly higher in GD patients carrying the risk genotypes of the two independent susceptibility SNPs (rs12101261 and rs179243) than in those carrying the protective genotypes after the patients had been treated with ATD for more than 1 year. These data may provide an insight to predicting the outcome of pTRAb + using genetic markers in GD patients.

In conclusion, the association of two independent susceptibility variants in TSHR, rs12101261 and rs179243, was refined in a large-scale study of the Chinese Han population. We also found that the rate of pTRAb + was significantly higher in the GD patients with the susceptible rs12101261 or rs179243 genotypes than in those carrying the protective genotypes, after the patients had been treated for more than 1 year. These findings indicate that the SNPs rs12101261 and rs179243 might be used as genetic markers to predict the outcome of pTRAb+ in GD patients. However, further functional studies are needed to investigate the mechanism underlying the induction of GD onset by the two SNPs.

\section{Supplementary data}

This is linked to the online version of the paper at http://dx.doi.org/10.1530/ EJE-13-0517.

\section{Declaration of interest}

The authors declare that there is no conflict of interest that could be perceived as prejudicing the impartiality of the research reported.

\section{Funding}

This study was funded by the National Natural Science Foundation of China (30971595, 30900503, 30971383, 81100553, 81000321, and 31171127), National Basic Research Program of China (973 Program) (2010CB529204 and 2012CB517604), Shanghai Science and Technology Committee
(10JC1410400), and Program for Graves' Disease Innovative Research Team of Shanghai Municipal Education Commission.

\section{Acknowledgements}

We thank all patients and control subjects for participating in this study.

\section{References}

1 Brix TH, Christensen K, Holm NV, Harvald B \& Hegedus L. A populationbased study of Graves' disease in Danish twins. Clinical Endocrinology 1998 48 397-400. (doi:10.1046/j.1365-2265.1998.00450.x)

2 Brix TH, Kyvik KO, Christensen K \& Hegedus L. Evidence for a major role of heredity in Graves' disease: a population-based study of two Danish twin cohorts. Journal of Clinical Endocrinology and Metabolism 200186 930-934. (doi:10.1210/jc.86.2.930)

3 Ueda H, Howson JM, Esposito L, Heward J, Snook H, Chamberlain G, Rainbow DB, Hunter KM, Smith AN, Di Genova G et al. Association of the T-cell regulatory gene CTLA4 with susceptibility to autoimmune disease. Nature 2003423 506-511. (doi:10.1038/nature01621)

4 Simmonds MJ \& Gough SC. The search for the genetic contribution to autoimmune thyroid disease: the never ending story? Brief Functional Genomics 201110 77-90. (doi:10.1093/bfgp/elq036)

5 Simmonds MJ, Howson JM, Heward JM, Cordell HJ, Foxall H, Carr-Smith J, Gibson SM, Walker N, Tomer Y, Franklyn JA et al. Regression mapping of association between the human leukocyte antigen region and Graves' disease. American Journal of Human Genetics 200576 157-163. (doi:10.1086/426947)

6 Simmonds MJ, Howson JM, Heward JM, Carr-Smith J, Franklyn JA, Todd JA \& Gough SC. A novel and major association of HLA-C in Graves' disease that eclipses the classical HLA-DRB1 effect. Human Molecular Genetics 200716 2149-2153. (doi:10.1093/hmg/ddm165)

7 Tomer Y. Genetic Susceptibility to Autoimmune Thyroid Disease: Past, Present, and Future. Thyroid 201020 715-725. (doi:10.1089/thy. 2010.1644)

8 Chu X, Pan CM, Zhao SX, Liang J, Gao GQ, Zhang XM, Yuan GY, Li CG Xue LQ, Shen $\mathrm{M}$ et al. A genome-wide association study identifies two new risk loci for Graves' disease. Nature Genetics 201143 897-901. (doi:10.1038/ng.898)

9 Zhao SX, Xue LQ, Liu W, Gu ZH, Pan CM, Yang SY, Zhan M, Wang HN, Liang J, Gao GQ et al. Robust evidence for five new Graves' disease risk loci from a staged genome-wide association analysis. Human Molecular Genetics 201322 3347-3362. (doi:10.1093/hmg/ddt183)

10 Szymanski K, Bednarczuk T, Krajewski P \& Ploski R. The replication of the association of the rs6832151 within chromosomal band 4p14 with Graves' disease in a Polish Caucasian population. Tissue Antigens 2012 79 380-383. (doi:10.1111/j.1399-0039.2012.01854.x)

11 Cooper JD, Simmonds MJ, Walker NM, Burren O, Brand OJ, Guo H, Wallace C, Stevens H, Coleman G, Franklyn JA et al. Seven newly identified loci for autoimmune thyroid disease. Human Molecular Genetics 201221 5202-5208. (doi:10.1093/hmg/dds357)

12 Allahabadia A, Heward JM, Mijovic C, Carr-Smith J, Daykin J, Cockram C, Barnett AH, Sheppard MC, Franklyn JA \& Gough SC. Lack of association between polymorphism of the thyrotropin receptor gene and Graves' disease in United Kingdom and Hong Kong Chinese patients: case control and family-based studies. Thyroid $1998 \mathbf{8}$ 777-780. (doi:10.1089/thy.1998.8.777)

13 Gabriel EM, Bergert ER, Grant CS, van Heerden JA, Thompson GB \& Morris JC. Germline polymorphism of codon 727 of human thyroidstimulating hormone receptor is associated with toxic multinodular goiter. Journal of Clinical Endocrinology and Metabolism 199984 3328-3335. (doi:10.1210/jc.84.9.3328)

14 Simanainen J, Kinch A, Westermark K, Winsa B, Bengtsson M, Schuppert F, Westermark B \& Heldin NE. Analysis of mutations in 
exon 1 of the human thyrotropin receptor gene: high frequency of the D36H and P52T polymorphic variants. Thyroid 1999 9 7-11.

(doi:10.1089/thy.1999.9.7)

15 Chistyakov DA, Savost'anov KV, Turakulov RI, Petunina NA, Trukhina LV, Kudinova AV, Balabolkin MI \& Nosikov VV. Complex association analysis of Graves' disease using a set of polymorphic markers. Molecular Genetics and Metabolism 200070 214-218. (doi:10.1006/mgme.2000.3007)

16 Kaczur V, Takacs M, Szalai C, Falus A, Nagy Z, Berencsi G \& Balazs C. Analysis of the genetic variability of the 1st (CCC/ACC, P52T) and the 10th exons (bp 1012-1704) of the TSH receptor gene in Graves' disease. European Journal of Immunogenetics 200027 17-23. (doi:10.1046/j.13652370.2000.00187.x)

17 Muhlberg T, Herrmann K, Joba W, Kirchberger M, Heberling HJ \& Heufelder AE. Lack of association of nonautoimmune hyperfunctioning thyroid disorders and a germline polymorphism of codon 727 of the human thyrotropin receptor in a European Caucasian population. Journal of Clinical Endocrinology and Metabolism 200085 2640-2643. (doi:10.1210/jc.85.8.2640)

18 Ban Y, Greenberg DA, Concepcion ES \& Tomer Y. A germline single nucleotide polymorphism at the intracellular domain of the human thyrotropin receptor does not have a major effect on the development of Graves' disease. Thyroid 200212 1079-1083. (doi:10.1089/ 105072502321085171)

19 Chistiakov DA, Savost'anov KV, Turakulov RI, Petunina N, Balabolkin MI \& Nosikov VV. Further studies of genetic susceptibility to Graves' disease in a Russian population. Medical Science Monitor 20028 CR180-CR184.

20 Ho SC, Goh SS \& Khoo DH. Association of Graves' disease with intragenic polymorphism of the thyrotropin receptor gene in a cohort of Singapore patients of multi-ethnic origins. Thyroid 200313 523-528. (doi:10.1089/105072503322238773)

21 Hiratani H, Bowden DW, Ikegami S, Shirasawa S, Shimizu A, Iwatani Y $\&$ Akamizu T. Multiple SNPs in intron 7 of thyrotropin receptor are associated with Graves' disease. Journal of Clinical Endocrinology and Metabolism 200590 2898-2903. (doi:10.1210/jc.2004-2148)

22 Brand OJ, Barrett JC, Simmonds MJ, Newby PR, McCabe CJ, Bruce CK, Kysela B, Carr-Smith JD, Brix T, Hunt PJ et al. Association of the thyroid stimulating hormone receptor gene (TSHR) with Graves' disease. Human Molecular Genetics 200918 1704-1713. (doi:10.1093/hmg/ ddp087)
23 Burton PR, Clayton DG, Cardon LR, Craddock N, Deloukas P, Duncanson A, Kwiatkowski DP, McCarthy MI, Ouwehand WH, Samani NJ et al. Association scan of 14,500 nonsynonymous SNPs in four diseases identifies autoimmunity variants. Nature Genetics 200739 1329-1337. (doi:10.1038/ng.2007.17)

24 Feldt-Rasmussen U, Schleusener H \& Carayon P. Meta-analysis evaluation of the impact of thyrotropin receptor antibodies on long term remission after medical therapy of Graves' disease. Journal of Clinical Endocrinology and Metabolism 199478 98-102. (doi:10.1210/jc.78.1.98)

25 Fukao A, Takamatsu J, Murakami Y, Sakane S, Miyauchi A, Kuma K, Hayashi S \& Hanafusa T. The relationship of psychological factors to the prognosis of hyperthyroidism in antithyroid drug-treated patients with Graves' disease. Clinical Endocrinology 200358 550-555. (doi:10.1046/j.1365-2265.2003.01625.x)

26 Aulchenko YS, Struchalin MV \& van Duijn CM. ProbABEL package for genome-wide association analysis of imputed data. BMC Bioinformatics 201011 134. (doi:10.1186/1471-2105-11-134)

27 Gustavsson B, Eklof C, Westermark K, Westermark B \& Heldin NE. Functional analysis of a variant of the thyrotropin receptor gene in a family with Graves' disease. Molecular and Cellular Endocrinology 1995 111 167-173. (doi:10.1016/0303-7207(95)03562-L)

28 Akamizu T, Sale MM, Rich SS, Hiratani H, Noh JY, Kanamoto N, Saijo M, Miyamoto Y, Saito Y, Nakao K et al. Association of autoimmune thyroid disease with microsatellite markers for the thyrotropin receptor gene and CTLA-4 in Japanese patients. Thyroid 200010 851-858. (doi:10.1089/thy.2000.10.851)

29 Dechairo BM, Zabaneh D, Collins J, Brand O, Dawson GJ, Green AP, Mackay I, Franklyn JA, Connell JM, Wass JA et al. Association of the TSHR gene with Graves' disease: the first disease specific locus. European Journal of Human Genetics 200513 1223-1230. (doi:10.1038/sj.ejhg. 5201485)

30 Schott M, Morgenthaler NG, Fritzen R, Feldkamp J, Willenberg HS, Scherbaum WA \& Seissler J. Levels of autoantibodies against human TSH receptor predict relapse of hyperthyroidism in Graves' disease. . Hormone and Metabolic Research 200436 92-96. (doi:10.1055/s-2004814217)

31 Eckstein A, Mann K, Kahaly GJ, Grussendorf M, Reiners C, Feldkamp J, Quadbeck B, Bockisch A \& Schott M. Role of TSH receptor autoantibodies for the diagnosis of Graves' disease and for the prediction of the course of hyperthyroidism and ophthalmopathy. Recommendations of the Thyroid Section of the German Society of Endocrinology. Medizinische Klinik 2009104 343-348. (doi:10.1007/s00063-009-1072-0)

Received 24 June 2013

Revised version received 4 October 2013

Accepted 21 October 2013 